OPEN ACCESS

Edited by:

Jianke Zhang,

Thomas Jefferson University,

United States

Reviewed by:

Donat Kögel,

Frankfurt University, Germany

Devrim Gozuacik,

Koç University, Turkey

Junichi Sadoshima,

University of Medicine and Dentistry

of New Jersey, United States

Jihoon Nah

Rutgers University, Newark,

United States

*Correspondence:

Lawrence M. Schwartz

Ims@bio.umass.edu

Specialty section:

This article was submitted to

Cell Death and Survival,

a section of the journal

Frontiers in Cell and Developmental

Biology

Received: 20 January 2021

Accepted: 17 March 2021

Published: 09 April 2021

Citation:

Schwartz LM (2021) Autophagic Cell Death During Development -

Ancient and Mysterious.

Front. Cell Dev. Biol. 9:656370. doi: 10.3389/fcell.2021.656370

\section{Autophagic Cell Death During Development - Ancient and Mysterious}

\author{
Lawrence M. Schwartz*
}

Department of Biology, Molecular and Cellular Biology Program, Morrill Science Center, University of Massachusetts, Amherst, MA, United States

While cell death is a normal and essential component of development and homeostasis, dysregulation of this process underlies most human diseases, including cancer, autoimmunity and neurodegeneration. The best characterized mechanism for cell death is apoptosis, although some cells die by a distinct process known as autophagy-dependent cell death (ADCD). Autophagy is mediated by the formation of double membrane vesicles that contain protein aggregates, damaged organelles like mitochondria, and bulk cytoplasm, which then fuse with lysosomes to degrade and recycle their contents. Autophagy is typically viewed as an adaptive process that allows cells to survive stresses like nutrient deprivation, although increasing evidence suggests that it may also mediate cell death during development and pathogenesis. An aggressive form of autophagy termed autosis has been described in cells following either ischemia/reperfusion injury or in response to autophagy-inducing proteins like TatBeclin 1. Despite an extensive literature on autophagic cell death in a variety of contexts, there are still fundamental gaps in our understanding of this process. As examples: Does autophagy directly kill cells and if so how? Is ADCD activated concurrently when cells are triggered to die via apoptosis? And is ADCD essentially a more protracted version of autosis or a distinct pathway? The goal of this mini-review is to summarize the field and to identify some of the major gaps in our knowledge. Understanding the molecular mechanisms that mediate ADCD will not only provide new insights into development, they may facilitate the creation of better tools for both the diagnostics and treatment of disease.

Keywords: programmed cell death, apoptosis, autophagy, autosis, necrosis, Tat-Beclin 1, lysosome

\section{INTRODUCTION}

The term programmed cell death (PCD) was coined by Lockshin and Williams (1965) to describe the precisely timed loss of skeletal muscles at the end of metamorphosis in moths. PCD plays many essential roles during both development and homeostasis. First, it can insure the presence of the appropriate number of cells within each tissue. A general rule of embryogenesis is that many more 
cells are produced in each lineage than are ultimately needed to support organogenesis (Raff, 1992). Cells that make a connection with appropriate partners receive a retrograde signal referred to as a trophic factor. These cells then upregulate survival programs and persist, while their unsuccessful neighbors die during a discrete window of development. The classical example of this process is the matching of motor neurons to the muscles they innervate (Hamburger, 1934). Second, targeted cell death can help sculpt the body, such as interdigital cell death in the developing limb bud to form the fingers (Saunders et al., 1962). Third, cell death can target tissues that have served an important function at one stage of development but are then no longer needed at a later stage, such as regression of the tadpole tail during amphibian metamorphosis (Smith and Tata, 1976). These "surplus" tissues represent valuable reservoirs of macromolecules that can be used to support metabolism and development. Fourth, PCD can be used to remove deleterious cells, such as the loss of self-reactive thymocytes during negative selection in the thymus (Surh and Sprent, 1994). Lastly, PCD functions as a normal component of homeostasis. For example, we lose approximately one million cells per minute throughout life, primarily in the hematopoietic system (Levine and Ucker, 2019). These cells are typically replaced by the products of stem cells, thus maintaining tissue homeostasis.

However, not all cell deaths serve a constructive role; it has been estimated that misregulation of this process may account for upwards of $70 \%$ of human disease (Reed, 2002). In some cases, inappropriate activation of PCD results in the loss of valuable and irreplaceable cells, thus compromising the tissue. This is the basis of essentially all neurodegenerative disorders like Alzheimer's and Parkinson's diseases. In contrast, the failure to delete defective but mitoticallycompetent cells allows for their clonal expansion, which serves as the basis of most cancers and all autoimmune diseases. Consequently, one of the major drivers of the field is the desire to identify interventions that rescue valuable but condemned cells, or alternatively, selectively target defective ones.

During development, the molecules or cell-cell interactions that trigger PCD typically are not inherently toxic but instead are physiological signals that can activate a range of downstream responses, one of which may be death. This is illustrated in amphibian metamorphosis, where a dramatic increase in the circulating levels of thyroxine simultaneously induces proliferation and differentiation in the developing limb anlagen and cell death in the tail (Yaoita, 2019). However, cell death can also be provoked by external signals that do not normally function during development, such as ionizing radiation or toxins. Previous debate in the field questioned whether these cell deaths can accurately be termed "programmed," since they do not occur in a temporally or spatially predictable manner. To address this issue, the term Accidental Cell Death (ACD) was introduced to identify deaths that are induced by exogenous insults, while the term Regulated Cell Death (RCD) was coined to capture those cell deaths that employ specific cellular machinery, independent of the upstream trigger (Galluzzi et al., 2018). Thus, while the loss of immature T cells during negative selection is a traditional example of $\mathrm{RCD}$, the killing of these same cells with ionizing radiation represents $\mathrm{ACD}$, even though they both lead to apoptosis (Lowe et al., 1993; Galluzzi et al., 2018). PCD is still an acceptable term for those deaths that occur as a normal component of development.

\section{CELL DEATH PROGRAMS}

Historically, three distinct cell death programs were recognized based on morphological criteria: type I (nuclear degeneration/apoptosis), type II (cytoplasmic vacuolization/autophagic cell death), and type III degeneration (cell rupture/necrosis) (Schweichel and Merker, 1973; Clarke, 1990) (The term "degeneration" in this context is archaic and not in current use). The intense scientific focus on cell death $(\sim 500,000$ citations in Pubmed by the start of 2021) has revealed that there are many more pathways that can mediate cell loss. In addition to the three listed above, other programs include: ferroptosis, pyroptosis, necroptosis, parthanatos, entosis, mitochondrial permeability transition (MPT)-driven necrosis, lysosomedependent cell death, NETotic cell death, oxytosis, and mitotic catastrophe (reviewed in Galluzzi et al., 2018; Tang et al., 2019; Nirmala and Lopus, 2020).

Necrosis (formerly "type III degeneration") was initially viewed as a passive process whereby mechanical, chemical, or osmotic insults result in cellular swelling, membrane disruption and subsequent lysis. More recently, several regulated forms of necrosis have been identified, including necroptosis, pyroptosis, parthanoptosis, NETosis, MPT-driven necrosis, oxytosis, and ferroptosis, which are all dependent upon specific signaling pathways within the cell (Degterev et al., 2005; Dixon et al., 2012; Vanden Berghe et al., 2014; Alu et al., 2020). Since cytoplasmic and nuclear constituents are highly inflammatory (collectively known as damage-associated molecular patterns or DAMPS), these deaths can play valuable roles by mobilizing the immune system to respond to an acute injury or infection (Zindel and Kubes, 2020). In contrast, as membrane integrity is maintained during apoptosis, dying cells are typically phagocytosed and degraded by neighboring cells or macrophages, thereby precluding immunological responses. This is obviously beneficial given the massive numbers of cells that die during development and homeostasis, since it would be disastrous for the organism to be in a chronic hyperinflammatory state. [It should be noted that secondary necrosis of apoptotic cells in vitro, which serves as the basis for some widely used cell death assays, including vital dye exclusion, is an artifact resulting from the absence of phagocytic cells (Krysko et al., 2008). The discarded apoptotic bodies run out of the ATP required to maintain membrane pumps and undergo necrosis].

The best understood cell death program is apoptosis (formerly type I degeneration). This is due in part to outstanding genetic models like the nematode Caenorhabditis elegans, where the key components of the program were identified via mutational 
analysis (Malin and Shaham, 2015). In addition, the availability of highly sensitive and reliable assays like TUNEL (DNA fragmentation) and Annexin $\mathrm{V}$ staining (phosphatidylserine externalization) allow for the easy detection of apoptotic cells both in vitro and in vivo (Galluzzi et al., 2009).

Apoptosis is typically driven by the activation of procaspases, a family of cysteine-aspartic proteases that cleave a vast range of cellular proteins (Julien and Wells, 2017). There are two main pathways for caspase activation- extrinsic and intrinsic (reviewed in Bedoui et al., 2020). Ligand binding to membrane death receptors like TRAIL and FAS lead to the formation of the Death-Inducing Signaling Complex (DISC), and the subsequent activation of pro-caspase-8, which then activates the downstream executioner pro-caspases-3 and 7. The intrinsic pathway is initiated when developmental or pathological signals lead to a shift in the balance between pro- and anti-apoptotic Bcl-2 family members that regulate the oligomerization of $\mathrm{BAX}$ and $\mathrm{BAK}$. These proteins can form a pore that mediates mitochondrial outer membrane permeabilization (MOMP) causing the release of cytochrome $c$ and other pro-apoptotic proteins like SMAC/DIABLO and apoptosis inducing factor (AIF) (Kalkavan and Green, 2018). Cytochrome $c$ and dATP bind to apoptotic protease activating factor 1 (APAF-1) to facilitate the formation of the apoptosome and the subsequent activation of the initiator caspase-9, and ultimately, the activation of executioner caspases. The extrinsic and intrinsic pathways can be coupled via caspase- 8 cleavage of the BH3-only protein Bid to form truncated Bid (tBid) (Li et al., 1998).

Much less is known about the third classic mechanism of cell death- Type II degeneration/autophagic cell death. The best characterized form of autophagy is macroautophagy, and the two terms are often used interchangeably (Other autophagic mechanisms also include microautophagy and chaperone-mediated autophagy). Autophagy is an evolutionarily ancient process that allows cells to survive starvation and other stresses by facilitating the sequestration and degradation of bulk cytoplasm, damaged organelles like mitochondria and protein aggregates (Hughes and Rusten, 2007; Ohsumi, 2014; Wanderoy et al., 2020). The recycled amino acids and fatty acids facilitate ATP production and other essential processes. The basal levels of autophagy can be upregulated under conditions of nutrient deprivation or loss of growth factors (Figure 1; reviewed in Cicchini et al., 2015). This leads to inhibition of mTorcl and/or the activation of 5' AMP-activated protein kinase (AMPK) and Class III phosphatidylinositol 3-kinase. These drive the formation of the phagophore, a crescent shaped double membrane structure generated from intracellular membranes. The elongation of the phagophore membrane is mediated by two ubiquitinlike enzymatic cascade (Atg12-Atg5 and LC3/Atg8), which facilitates the phosphatidylethanolamine-lipidated LC3 (ATG8) and it's recruitment to the membrane (reviewed in Martens and Fracchiolla, 2020). The phagophore then circularizes to form the autophagosome, which then fuses with lysosomes, resulting in the creation of the autolysosomes, where the trapped contents are degraded.

\section{AUTOPHAGY-DEPENDENT CELL DEATH}

Given that autophagy can be an adaptive response that helps cells survive adverse conditions, there is still a lack of clarity of if and how autophagy is part of a directed killing program. In fact, Kroemer and Levine (2008) have suggested that the term "autophagic cell death" is a misnomer and that a more appropriate descriptor might be "cell death with autophagy". Consequently, in order to determine that cells die via autophagydependent cell death (ADCD), it has been proposed that three criteria must be met: (1) other forms of cell death have been excluded; (2) there is an increase in autophagic flux; and (3) genetic or pharmacological blockade of autophagy rescues the cell (Shen and Codogno, 2011; Galluzzi et al., 2018). These strict requirements have been met in only a small number of studies, so the number of bona fide ADCD systems is likely more modest than reflected by a Pubmed search with "autophagic cell death" as the search term (Bialik et al., 2018). Nevertheless, there are several genetic models where an absolute requirement for the autophagic machinery has been shown to be essential for cell death. From a phylogenetic perspective, perhaps the most ancient example is found in the free-living social ameba Dictyostelium discoideum (Cornillon et al., 1994). While "Dicty" live solitary lives in the soil, the loss of food triggers a signal transduction cascade that induces the cells to aggregate and form a stalk with a spore-containing fruiting body at the top. As differentiation proceeds, the stalk cells die by an autophagydependent pathway. Indeed, targeting the autophagy-related gene-1 (Atg1) blocks death and the cells instead default to necrosis (Kosta et al., 2004).

The fruit fly Drosophila melanogaster also provides several well-characterized examples of autophagic cell death during metamorphosis. The loss of the salivary glands is dependent on both apoptosis and autophagy, as genetic interference with either of these pathways represses but does not prevent cell death (Martin and Baehrecke, 2004; Berry and Baehrecke, 2007). In contrast, the death of the gut appears to require just autophagy, as genetic inactivation or knockdown Atg1, Atg2 or Atg18 severely delays these deaths (Denton et al., 2009, 2012). Midgut death in flies is recognized as the clearest and most convincing example of ADCD during development.

There are a number of studies supporting the existence of ADCD in mammals, most notably in terminally differentiated cells like muscles and neurons, and some cancers (Linder and Kögel, 2019). Examples include the loss of neurons in neurodegenerative disorders like Alzheimer's disease (Nixon et al., 2005; Bredesen, 2008), spinal cord injury (Kanno et al., 2011), MPTP-induced cytotoxicity (Zhao et al., 2019), and neonatal hypoxia-ischemia following asphyxia (Ginet et al., 2014). Perhaps the best supported examples have focused on the death of hippocampal stem cells following insulin withdrawal in vitro (Yu et al., 2008) or following chronic stress in vivo (Jung et al., 2020).

Cells that are null for the pro-apoptotic proteins BAK and BAX, and thus incapable of initiating apoptosis provide intriguing insights into ADCD (Shimizu et al., 2004; Arakawa et al., 2017). When mouse embryonic fibroblasts from 


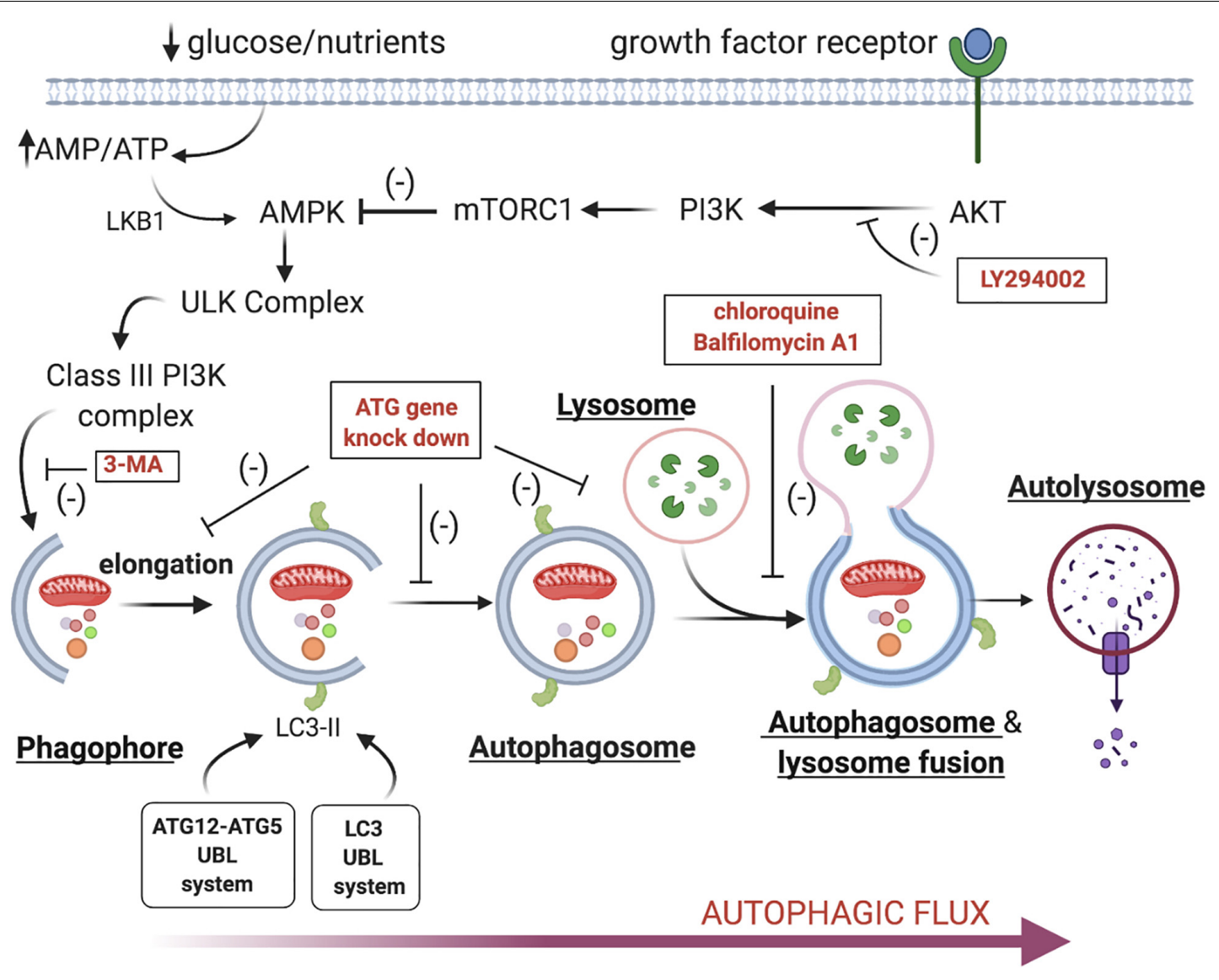

FIGURE 1 | Signal transduction cascade regulating autophagy. Loss of growth factor stimulation or nutritional inputs like glucose lead to the activation of the ULK complex and the subsequent activation of the Class III PI3 Kinase complex. This drives phagophore assembly and the subsequent recruitment of cellular constituents like damaged mitochondria, protein aggregates and bulk cytoplasm. The phagophore elongates and circularizes to form the autophagosome, which then docks with lysosomes. The fusion of these two organelles leads to autolysosome formation and the destruction of the enclosed cargo via lysosomal hydrolases. [It should be noted that this is distinct from lysosome-mediated cell death in which lysosome rupture and release degradative enzymes like cathepsins (reviewed in Wang et al., 2018)]. Several inhibitors or genetic interventions are available that can block autophagy at key regulatory points (labeled red in boxes)

(Vakifahmetoglu-Norberg et al., 2015).

$\mathrm{Bax}^{-/-} \mathrm{Bak} \mathrm{k}^{-/-}$mice were treated with the potent apoptosis inducers etoposide or staurosporine, they initiated ADCD (Shimizu et al., 2004). Concurrent inactivation of Atg5 in $B a x^{-/-} \mathrm{Bak}^{-/-}$mice restricted both apoptosis and autophagy. This led to the retention of otherwise condemned cells, including interdigital cells in the limb buds, thymocytes, and some neurons (Arakawa et al., 2017). These data provide strong evidence autophagy is required for PCD during normal mammalian development.

In 2013 a novel form of autophagic cell death termed "autosis" was described in cells that are treated with Tat-Beclin 1, a potent cell-permeant autophagy inducer (Liu et al., 2013; Liu and Levine, 2015). Autotic cells display a dramatic increase in the number of autophagic vesicles and empty vesicles within the cytoplasm. Over time the mitochondria become electron dense, the perinuclear space balloons as the inner and outer nuclear membranes separate, and the nucleus displays concavity. A similar set of morphological changes can be observed in vivo in cardiomyocytes in adult mice and rat neonatal neurons following ischemia/reperfusion injury (Liu et al., 2013; Nah et al., 2016, 2020), a process that is clinically relevant to heart attacks and strokes respectively. Autosis can be inhibited genetically by inactivating essential autophagy genes (e.g., Beclin1 and Atg7) or pharmacologically with anti-autophagic drugs that block phagophore assembly (e.g., 3-methyladenine) but not lysosome fusion (e.g., Bafilomycin A1) (Figure 1; Liu et al., 2013). In a high throughput screen, it was also found that inhibitors of $\mathrm{Na}^{+}, \mathrm{K}^{+}$adenosine triphosphatase (ATPase) like the cardiac glycosides ouabain and digoxin can also inhibit autosis, which is diagnostic for autosis (Liu et al., 2013; Nah et al., 2020).

\section{UNANSWERED QUESTIONS}

While the data presented in this review provides an overview of cell death in general, and ADCD during development in particular, there are still several major gaps in our fundamental understanding of this process (Lindqvist et al., 2015; 


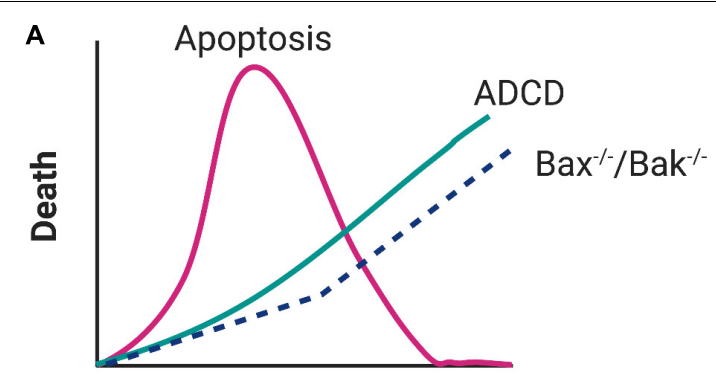

Time
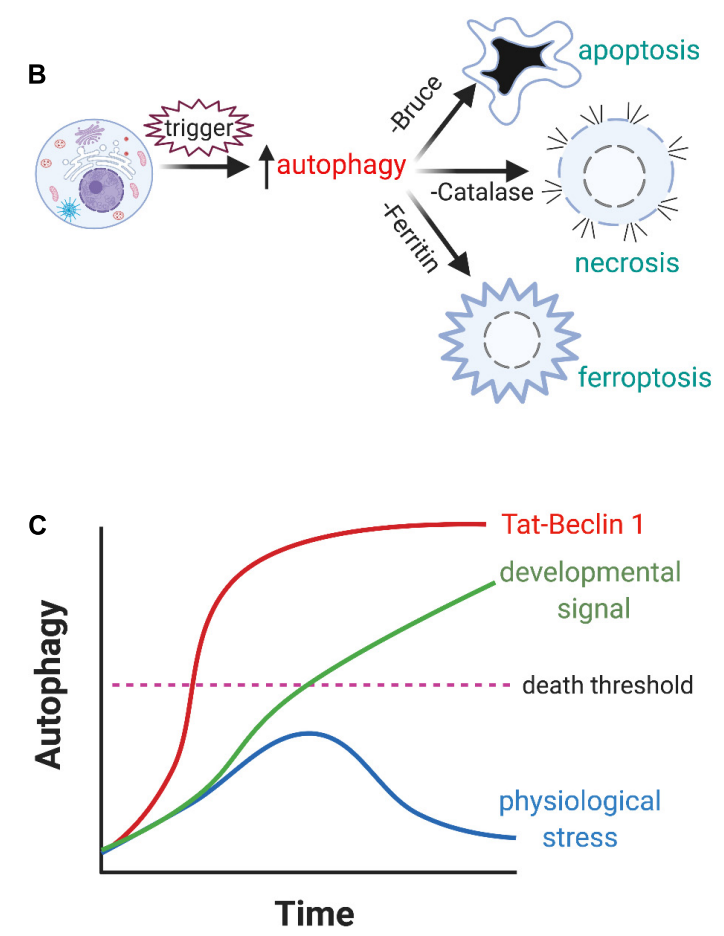

FIGURE 2 | Proposed ADCD pathways. (A) Both apoptosis and ADCD are triggered concurrently but the speed of apoptosis masks the involvement of ADCD. Blockage of apoptosis in Bax ${ }^{-/-} \mathrm{Bak}^{-/-}$cells allows autophagy to progress to the death of the cell. (B) Autophagy derepresses other cell death programs. By selectively targeting key survival proteins, compromised cells are able to activate non-autophagic cell death programs. (C) Treating cells with potent autophagy inducers like Tat-Beclin 1 results in the rapid demise of the cells (above the dashed "death threshold" line). If a cell is subjected to mild stresses, it transiently upregulates autophagy, which helps it survive the insult. However, when the cell is exposed to a cell death inducer during development, autophagy is driven past the point of no return and the cell dies.

Bialik et al., 2018). Some of the key questions that remain unresolved are discussed below.

1. Is ADCD widespread but masked by apoptosis?: Some insight into this question is provided from the study of cells from $\mathrm{Bax}^{-/-} \mathrm{Bak^{-/- }}$ mice, where condemned cells are precluded from undergoing apoptosis and default to
ADCD (Shimizu et al., 2004; Arakawa et al., 2017). There are two possible interpretations of these data. The first is that apoptosis and ADCD are triggered concurrently, but $\mathrm{ADCD}$ is masked by the much faster apoptosis program (Figure 2A). Alternatively, when cells are unable to activate their primary cell death program, there is compensatory initiation of another one to ensure that condemned cells are removed. Given the complicated cross-talk between autophagic and apoptotic proteins, such as Beclin 1 and Bcl-2 (Xu and Qin, 2019), this process is feasible but challenging to resolve (reviewed in Tsapras and Nezis, 2017).

2. Does autophagy trigger cell death indirectly? Autophagy can selectively target the destruction of key survival proteins like the inhibitor of apoptosis protein (IAP) BRUCE (which prevents pro-caspase activation) (Nezis et al., 2010), catalase (which blocks free radical generation) (Yu et al., 2006), and ferritin (which sequesters iron) (Gao et al., 2016). Loss of these survival proteins subsequently facilitates cell death (Figure 2B). However, instead of dying with an autophagic morphology, these condemned cells die by apoptosis, necrosis, or ferroptosis respectively. Consequently, while autophagy can derepress cell death, it does not meet the three requirements of ADCD in that the actual death process is mediated by a different program.

3. Is $\mathrm{ADCD}$ a less aggressive form of autosis? A number of studies have shown a direct correlation between autosis and cell death. Indeed, inhibition of autosis with cardiac glycosides can rescue these otherwise condemned cells (Liu et al., 2013; Nah et al., 2020). One possibility is that autosis is just a more aggressive version of the same program that mediates ADCD during development (Figure 2C). In support of this hypothesis, knockdown of upstream autophagy regulators (beclin1, Atg13, and Atg14) can block Tat-Beclin 1-induced cell death (Liu et al., 2013). An overlap between autosis and ADCD would offer mechanistic insights into the cell death process since autosis is accompanied by damage to organelles like mitochondria and the nucleus (Liu et al., 2013). In addition, the aggressive production of intracellular vacuoles during autosis is associated with the breakdown of the Golgi complex and the endoplasmic reticulum, which depletes intracellular membranes, something that is seen in some examples of ADCD (Liu and Levine, 2015). This may explain why Bafilomycin A1, which blocks lysosome fusion to autophagosomes, does not inhibit autosis (Liu et al., 2013). In the case of ischemia/reperfusion injury there is also a dramatic increase in the production of destructive free radicals (Wu et al., 2018). Collectively, these insults likely bring about the demise of the cell. However the relationship between $\mathrm{ADCD}$ and autosis is unclear. All of the examples of autosis described thus far result from pathological drivers of autophagy, like Tat-Beclin 1 or ischemia/reperfusion injury, rather than by physiological changes within a developmental context. It should also be noted that the morphologies associated with ADCD and 
autosis are distinct, although this may reflect the faster kinetics or intensity of autosis relative to the more protracted time course of ADCD. Clearly this is an area that warrants further investigation.

\section{CONCLUSION}

While it has not been demonstrated unambiguously that autophagy drives cell death during development (Kroemer and Levine, 2008), there is increasing evidence that ADCD is a bonafide cell death program. Nevertheless, there are still major gaps in our understanding of this mechanism in development and pathogenesis. Resolving these issues will not only advance our fundamental understanding of an ancient cellular process that is relevant to developmental biology and homeostasis, it may provide insights into the diagnostics and/or therapeutics for disease. In particular, ADCD appears to be an important

\section{REFERENCES}

Alu, A., Han, X., Ma, X., Wu, M., Wei, Y., and Wei, X. (2020). The role of lysosome in regulated necrosis. Acta Pharm. Sin. B. 10, 1880-1903. doi: 10.1016/j.apsb. 2020.07.003

Arakawa, S., Tsujioka, M., Yoshida, T., Tajima-Sakurai, H., Nishida, Y., Matsuoka, Y., et al. (2017). Role of Atg5-dependent cell death in the embryonic development of bax/bak double-knockout mice. Cell Death Differ. 24, 15981608. doi: $10.1038 / \mathrm{cdd} .2017 .84$

Bedoui, S., Herold, M. J., and Strasser, A. (2020). Emerging connectivity of programmed cell death pathways and its physiological implications. Nat. Rev. Mol. Cell Biol. 21, 678-695. doi: 10.1038/s41580-020-0270-8

Berry, D. L., and Baehrecke, E. H. (2007). Growth arrest and autophagy are required for salivary gland cell degradation in drosophila. Cell 131, 1137-1148. doi: $10.1016 /$ j.cell.2007.10.048

Bialik, S., Dasari, S. K., and Kimchi, A. (2018). Autophagy-dependent cell death - where, how and why a cell eats itself to death. J. Cell Sci. 131:jcs215152. doi: $10.1242 /$ jcs. 215152

Bredesen, D. E. (2008). Programmed cell death mechanisms in neurological disease. Curr. Mol. Med. 8, 173-186. doi: 10.2174/156652408784221315

Cicchini, M., Karantza, V., and Xia, B. (2015). Molecular pathways: autophagy in cancer-a matter of timing and context. Clin. Cancer Res. 21, 498-504. doi: 10.1158/1078-0432.CCR-13-2438

Clarke, P. G. (1990). Developmental cell death: morphological diversity and multiple mechanisms. Anat. Embryol. 181, 195-213.

Cornillon, S., Foa, C., Davoust, J., Buonavista, N., Gross, J. D., and Golstein, P. (1994). Programmed cell death in dictyostelium. J. Cell Sci. 107, 2691-2704.

Degterev, A., Huang, Z., Boyce, M., Li, Y., Jagtap, P., Mizushima, N., et al. (2005). Chemical inhibitor of nonapoptotic cell death with therapeutic potential for ischemic brain injury. Nat. Chem. Biol. 1, 112-119. doi: 10.1038/nchembio711

Denton, D., Chang, T. K., Nicolson, S., Shravage, B., Simin, R., Baehrecke, E. H., et al. (2012). Relationship between growth arrest and autophagy in midgut programmed cell death in drosophila. Cell Death Differ. 19, 1299-1307. doi: $10.1038 /$ cdd 2012.43

Denton, D., Shravage, B., Simin, R., Mills, K., Berry, D. L., Baehrecke, E. H., et al. (2009). Autophagy, not apoptosis, is essential for midgut cell death in drosophila. Curr. Biol. 19, 1741-1746. doi: 10.1016/j.cub.2009.08.042

Dixon, S. J., Lemberg, K. M., Lamprecht, M. R., Skouta, R., Zaitsev, E. M., Gleason, C. E., et al. (2012). Ferroptosis: an iron-dependent form of nonapoptotic cell death. Cell 149, 1060-1072. doi: 10.1016/j.cell.2012.03.042

Galluzzi, L., Aaronson, S. A., Abrams, J., Alnemri, E. S., Andrews, D. W., Baehrecke, E. H., et al. (2009). Guidelines for the use and interpretation of assays for monitoring cell death in higher eukaryotes. Cell Death Differ. 16, 1093-1107. doi: $10.1038 / \mathrm{cdd} .2009 .44$ process in neurodegeneration, cancer, and cardiology. The field will benefit greatly from the identification of the kinds of markers and inhibitors that have propelled the study of apoptosis.

\section{AUTHOR CONTRIBUTIONS}

LS wrote the manuscript and prepared the figures.

\section{ACKNOWLEDGMENTS}

The author would like to thank Drs. Amanda Woerman and Rachid Skouta for a critical reading of the manuscript, Ms. Christine Brown for technical assistance, and the Eugene M. and Ronnie Isenberg Professorship Endowment for support. The figures were created with BioRender.

Galluzzi, L., Vitale, I., Aaronson, S. A., Abrams, J. M., Adam, D., Agostinis, P., et al. (2018). Molecular mechanisms of cell death: recommendations of the nomenclature committee on cell death 2018. Cell Death Differ. 25, 486-541. doi: 10.1038/s41418-017-0012-4

Gao, M., Monian, P., Pan, Q., Zhang, W., Xiang, J., and Jiang, X. (2016). Ferroptosis is an autophagic cell death process. Cell Res. 26, 1021-1032. doi: 10.1038/cr. 2016.95

Ginet, V., Pittet, M. P., Rummel, C., Osterheld, M. C., Meuli, R., Clarke, P. G., et al. (2014). Dying neurons in thalamus of asphyxiated term newborns and rats are autophagic. Ann. Neurol. 76, 695-711. doi: 10.1002/ana.24257

Hamburger, V. (1934). The effects of wing bud extirpation on the development of the central nervous system in chick embryos. J. Exp. Zool. 68, 449-494. doi: 10.1002/jez.1400680305

Hughes, T., and Rusten, T. E. (2007). Origin and evolution of self-consumption: autophagy. Adv. Exp. Med. Biol. 607, 111-118. doi: 10.1007/978-0-387-74 021-8_9

Julien, O., and Wells, J. A. (2017). Caspases and their substrates. Cell Death Differ. 24, 1380-1389. doi: 10.1038/cdd.2017.44

Jung, S., Choe, S., Woo, H., Jeong, H., An, H. K., Moon, H., et al. (2020). Autophagic death of neural stem cells mediates chronic stress-induced decline of adult hippocampal neurogenesis and cognitive deficits. Autophagy 16, 512530. doi: 10.1080/15548627.2019.1630222

Kalkavan, H., and Green, D. R. (2018). MOMP, cell suicide as a BCL-2 family business. Cell Death Differ. 25, 46-55. doi: 10.1038/cdd.2017.179

Kanno, H., Ozawa, H., Sekiguchi, A., Yamaya, S., and Itoi, E. (2011). Induction of autophagy and autophagic cell death in damaged neural tissue after acute spinal cord injury in mice. Spine 36:1427. doi: 10.1097/BRS.0b013e3182028c3a

Kosta, A., Roisin-Bouffay, C., Luciani, M. F., Otto, G. P., Kessin, R. H., and Golstein, P. (2004). Autophagy gene disruption reveals a non-vacuolar cell death pathway in dictyostelium. J. Biol. Chem. 279, 48404-48409. doi: 10.1074/ jbc.M408924200

Kroemer, G., and Levine, B. (2008). Autophagic cell death: the story of a misnomer. Nat. Rev. Mol. Cell. Biol. 9, 1004-1010. doi: 10.1038/nrm2529

Krysko, D. V., Vanden Berghe, T., D’Herde, K., and Vandenabeele, P. (2008). Apoptosis and necrosis: detection, discrimination and phagocytosis. Methods 44, 205-221. doi: 10.1016/j.ymeth.2007.12.001

Levine, J. S., and Ucker, D. S. (2019). Voices from the dead: the complex vocabulary and intricate grammar of dead cells. Adv. Protein Chem. Struct. Biol. 116, 1-90. doi: 10.1016/bs.apcsb.2019.02.004

Li, H., Zhu, H., Xu, C. J., and Yuan, J. (1998). Cleavage of BID by caspase 8 mediates the mitochondrial damage in the fas pathway of apoptosis. Cell 94, 491-501. doi: 10.1016/s0092-8674(00)81590- 1

Linder, B., and Kögel, D. (2019). Autophagy in cancer cell death. Biology 8:82. doi: 10.3390/biology 8040082 
Lindqvist, L. M., Simon, A. K., and Baehrecke, E. H. (2015). Current questions and possible controversies in autophagy. Cell Death Discov. 1:15036. doi: 10.1038/ cddiscovery.2015.36

Liu, Y., and Levine, B. (2015). Autosis and autophagic cell death: the dark side of autophagy. Cell Death Differ. 22, 367-376. doi: 10.1038/cdd.2014.143

Liu, Y., Shoji-Kawata, S., Sumpter, R. M., Wei, Y., Ginet, V., Zhang, L., et al. (2013). Autosis is a $\mathrm{Na}+, \mathrm{K}+-\mathrm{ATPase}-$ regulated form of cell death triggered by autophagy-inducing peptides, starvation, and hypoxia-ischemia. Proc. Natl. Acad. Sci. U. S. A. 110, 20364-20371. doi: 10.1073/pnas.1319661110

Lockshin, R. A., and Williams, C. M. (1965). Programmed cell death-I. cytology of degeneration in the intersegmental muscles of the pernyi silkmoth. J. Insect Physiol. 11, 123-133. doi: 10.1016/0022-1910(65)90099-5

Lowe, S. W., Schmitt, E. M., Smith, S. W., Osborne, B. A., and Jacks, T. (1993). P53 is required for radiation-induced apoptosis in mouse thymocytes. Nature 362, 847-849. doi: 10.1038/362847a0

Malin, J. Z., and Shaham, S. (2015). Cell death in C. elegans development. Curr. Top. Dev. Biol. 114, 1-42. doi: 10.1016/bs.ctdb.2015.07.018

Martens, S., and Fracchiolla, D. (2020). Activation and targeting of ATG8 protein lipidation. Cell Discov. 6:23. doi: 10.1038/s41421-020-0155-1

Martin, D. N., and Baehrecke, E. H. (2004). Caspases function in autophagic programmed cell death in drosophila. Development 131, 275-284. doi: 10.1242/ dev.00933

Nah, J., Fernandez, A. F., Kitsis, R. N., Levine, B., and Sadoshima, J. (2016). Does autophagy mediate cardiac myocyte death during stress? Circ. Res. 119, 893-895. doi: 10.1161/CIRCRESAHA.116.309765

Nah, J., Zhai, P., Huang, C. Y., Fernandez, A. F., Mareedu, S., Levine, B., et al. (2020). Upregulation of rubicon promotes autosis during myocardial ischemia/reperfusion injury. J. Clin. Invest. 130, 2978-2991. doi: 10.1172/ JCI132366

Nezis, I. P., Shravage, B. V., Sagona, A. P., Johansen, T., Baehrecke, E. H., and Stenmark, H. (2010). Autophagy as a trigger for cell death: autophagic degradation of inhibitor of apoptosis dBruce controls DNA fragmentation during late oogenesis in drosophila. Autophagy 6, 1214-1215. doi: 10.4161/auto. 6.8.13694

Nirmala, J. G., and Lopus, M. (2020). Cell death mechanisms in eukaryotes. Cell Biol. Toxicol. 36, 145-164. doi: 10.1007/s10565-019-09496-2

Nixon, R. A., Wegiel, J., Kumar, A., Yu, W. H., Peterhoff, C., Cataldo, A., et al. (2005). Extensive involvement of autophagy in alzheimer disease: an immunoelectron microscopy study. J. Neuropathol. Exp. Neurol. 64, 113-122. doi: 10. 1093/jnen/64.2.113

Ohsumi, Y. J. W. (2014). Historical landmarks of autophagy research. Cell Res. 24, 9-23. doi: 10.1038/cr.2013.169

Raff, M. C. (1992). Social controls on cell survival and cell death. Nature 356, 397-400. doi: 10.1038/356397a0

Reed, J. C. (2002). Apoptosis-based therapies. Nat. Rev. Drug Discov. 1, 111-121. doi: $10.1038 / \mathrm{nrd} 726$

Saunders, J. W. Jr., Gasseling, M. T., and Saunders, L. C. (1962). Cellular death in morphogenesis of the avian wing. Dev. Biol. 5, 147-178. doi: 10.1016/00121606(62)90008-8

Schweichel, J. U., and Merker, H. J. (1973). The morphology of various types of cell death in prenatal tissues. Teratology 7, 253-266. doi: 10.1002/tera.1420070306

Shen, H. M., and Codogno, P. (2011). Autophagic cell death: loch ness monster or endangered species? Autophagy 7, 457-465. doi: 10.4161/auto.7.5.14226

Shimizu, S., Kanaseki, T., Mizushima, N., Mizuta, T., Arakawa-Kobayashi, S., Thompson, C. B., et al. (2004). Role of bcl-2 family proteins in a non-apoptotic programmed cell death dependent on autophagy genes. Nat. Cell Biol. 6, 1221-1228. doi: 10.1038/ncb1192

Smith, K. B., and Tata, J. R. (1976). Cell death. are new proteins synthesized during hormone-induced tadpole tail regression? Exp. Cell Res. 100, 129-146. doi: 10.1016/0014-4827(76)90335-9

Surh, C. D., and Sprent, J. (1994). T-cell apoptosis detected in situ during positive and negative selection in the thymus. Nature 372, 100-103. doi: 10.1038/ 372100a0

Tang, D., Kang, R., Berghe, T. V., Vandenabeele, P., and Kroemer, G. (2019). The molecular machinery of regulated cell death. Cell Res. 29, 347-364. doi: 10.1038/s41422-019-0164-5

Tsapras, P., and Nezis, I. (2017). Caspase involvement in autophagy. Cell Death Differ. 24, 1369-1379. doi: 10.1038/cdd.2017.43

Vakifahmetoglu-Norberg, H., Xia, H. G., and Yuan, J. (2015). Pharmacologic agents targeting autophagy. J. Clin. Invest. 125, 5-13. doi: 10.1172/JCI73937

Vanden Berghe, T., Linkermann, A., Jouan-Lanhouet, S., Walczak, H., and Vandenabeele, P. (2014). Regulated necrosis: the expanding network of nonapoptotic cell death pathways. Nat. Rev. Mol. Cell Biol. 15, 135-147. doi: 10. 1038/nrm 3737

Wanderoy, S., Hees, J. T., Klesse, R., Edlich, F., and Harbauer, A. B. (2020). Kill one or kill the many: interplay between mitophagy and apoptosis. Biol. Chem. 402, 73-88. doi: 10.1515/hsz-2020-0231

Wang, F., Gómez-Sintes, R., and Boya, P. (2018). Lysosomal membrane permeabilization and cell death. Traffic 19, 918-931. doi: 10.1111/tra.12613

Wu, M. Y., Yiang, G. T., Liao, W. T., Tsai, A. P., Cheng, Y. L., Cheng, P. W., et al. (2018). Current mechanistic concepts in ischemia and reperfusion injury. Cell Physiol. Biochem. 46, 1650-1667. doi: 10.1159/000489241

Xu, H. D., and Qin, Z. H. (2019). Beclin 1, bcl-2 and autophagy. Adv. Exp. Med. Biol. 1206, 109-126. doi: 10.1007/978-981-15-0602-4_5

Yaoita, Y. (2019). Tail resorption during metamorphosis in xenopus tadpoles. Front. Endocrinol. 10:143. doi: 10.3389/fendo.2019.00143

Yu, L., Wan, F., Dutta, S., Welsh, S., Liu, Z., Freundt, E., et al. (2006). Autophagic programmed cell death by selective catalase degradation. Proc. Natl. Acad. Sci. U. S. A. 103, 4952-4957. doi: 10.1073/pnas.0511288103

Yu, S. W., Baek, S. H., Brennan, R. T., Bradley, C. J., Park, S. K., Lee, Y. S., et al. (2008). Autophagic death of adult hippocampal neural stem cells following insulin withdrawal. Stem Cells 26, 2602-2610. doi: 10.1634/stemcells.2008-0153

Zhao, M., Chen, J., Mao, K., She, H., Ren, Y., Gui, C., et al. (2019). Mitochondrial calcium dysfunction contributes to autophagic cell death induced by $\mathrm{MPP}(+)$ via AMPK pathway. Biochem. Biophys. Res. Commun. 509, 390-394. doi: 10. 1016/j.bbrc.2018.12.148

Zindel, J., and Kubes, P. (2020). DAMPs, PAMPs, and LAMPs in immunity and sterile inflammation. Annu. Rev. Pathol. 15, 493-518. doi: 10.1146/annurevpathmechdis-012419-032847

Conflict of Interest: The author declares that the research was conducted in the absence of any commercial or financial relationships that could be construed as a potential conflict of interest.

Copyright (C) 2021 Schwartz. This is an open-access article distributed under the terms of the Creative Commons Attribution License (CC BY). The use, distribution or reproduction in other forums is permitted, provided the original author(s) and the copyright owner(s) are credited and that the original publication in this journal is cited, in accordance with accepted academic practice. No use, distribution or reproduction is permitted which does not comply with these terms. 